\title{
ANEURYSMAL DILATATION OF THE LEFT AURICLE WITH EROSION OF THE SPINE
}

BY

\author{
H. ASHWORTH AND A. MORGAN JONES \\ From the Cardiographic Department, Manchester Royal Infirmary \\ Received May 19, 1946
}

Although aneurysmal dilatation of the left auricle, due to mitral stenosis, is not uncommon, the following case is unusual for the enlarged auricle eroded the vertebral column, causing severe pain in the right chest, and became adherent to the right chest wall, leading to systolic retraction in the right axilla.

\section{CASE RECORD}

In August, 1945, a single woman, aged 38, employed in clerical work, was admitted to the Manchester Royal Infirmary, under the care of Dr. Crighton Bramwell, on account of severe pain in the right chest of four years' duration. Apart from scarlet fever and an antral operation in childhood, her health had been good until the age of 22 when she developed rheumatic fever and was treated in hospital for five months. She was subsequently troubled by occasional faintness and palpitation but carried on her occupation as a clerk, with little time off work owing to ill-health, until the age of 26 when she had an attack of lobar pneumonia. She never again resumed her occupation and became unable to undertake household duties owing

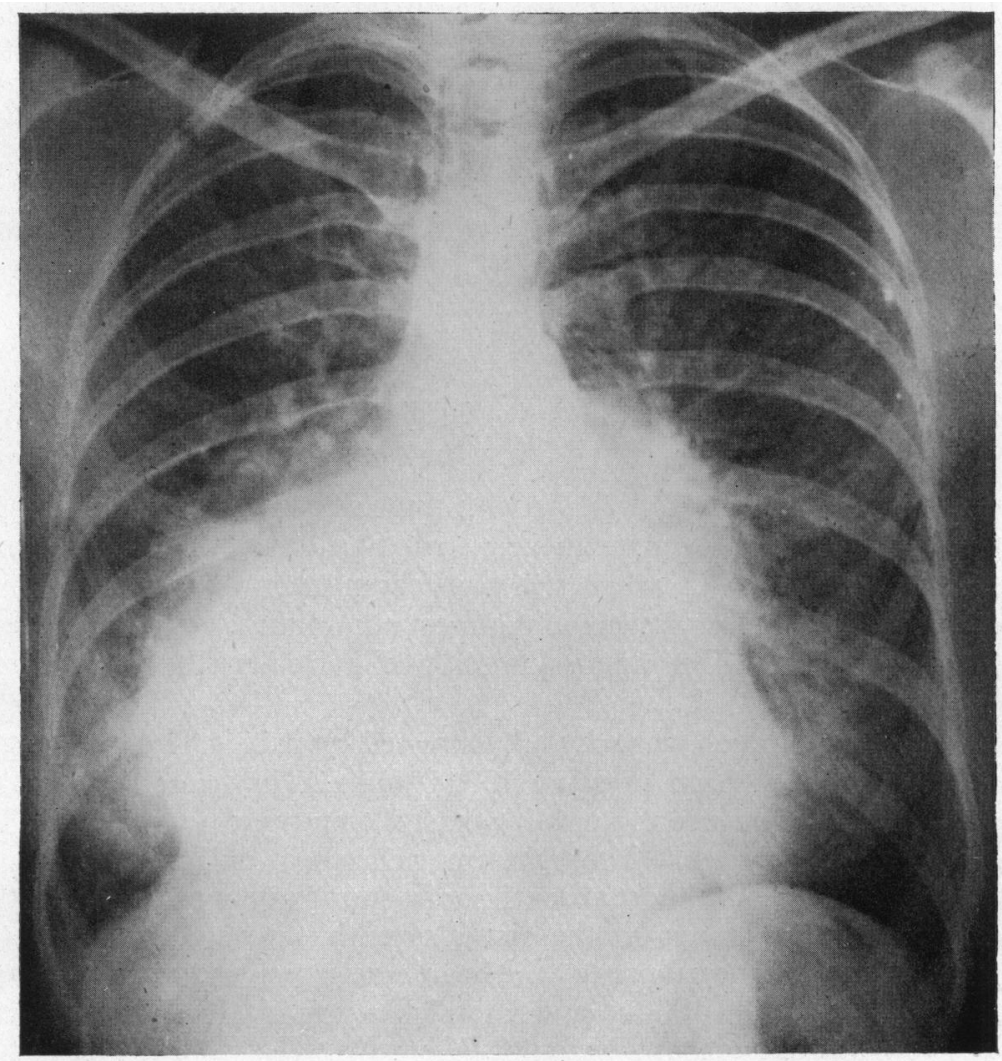

FIG. 1.-Teleradiogram, 1943. Anterior view, showing great dilatation of left auricle to the right. 
to severe dyspnoea on exertion. Aged 34, she began to suffer from gripping pain starting at the inferior angle of the right scapula and radiating round the chest to the right axilla. This occurred on exertion, on swallowing, or when her stomach was distended with food, and was relieved by rest and sometimes by alkalis. In July 1943, when aged 36, she was seen by Dr. Bramwell on account of this pain, and was found to be suffering from severe mitral stenosis with gross cardiac enlargement and uncontrolled auricular fibrillation. On cardioscopy, the left auricle was grossly dilated (Fig. 1 and 2). She was admitted to the Manchester Royal Infirmary,

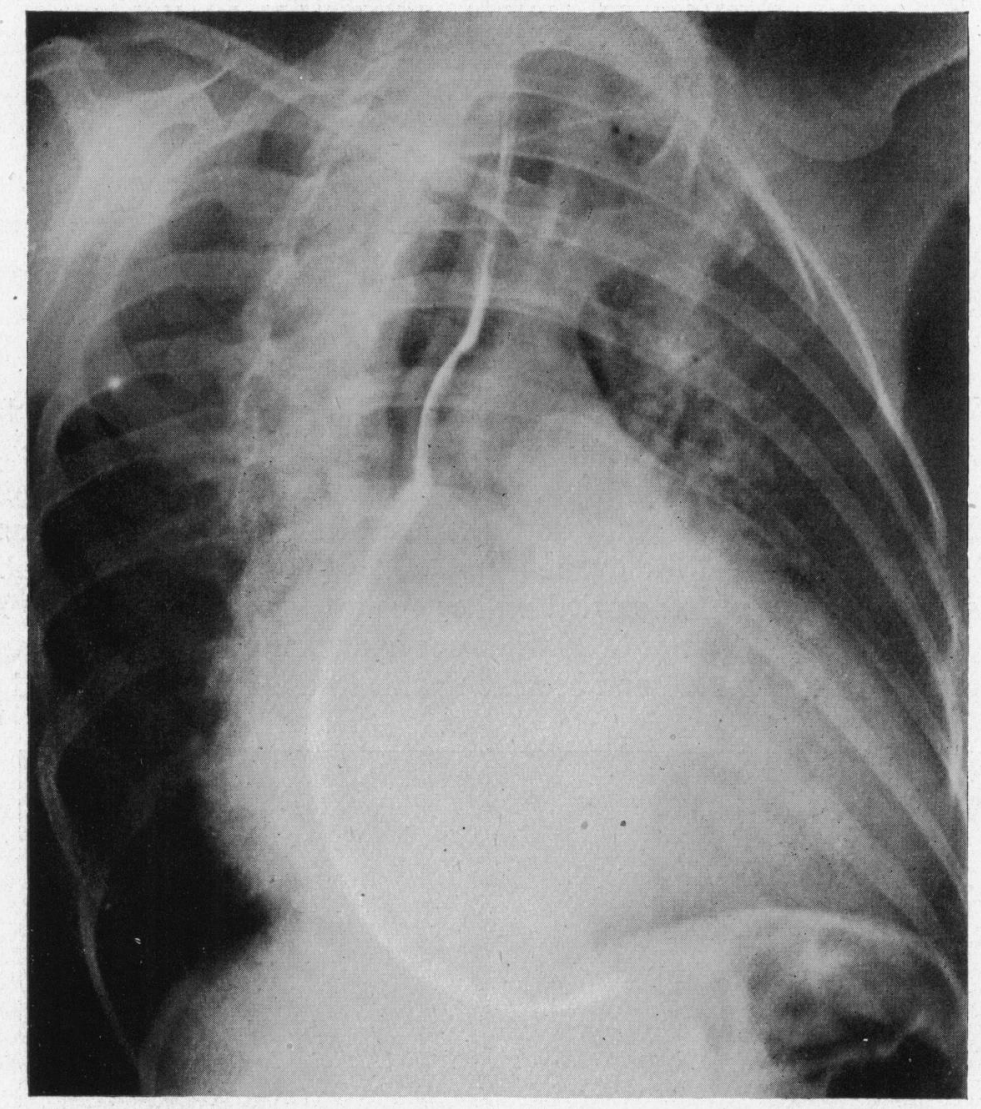

FIG. 2.-Right anterior (I) oblique radiogram, 1943, showing displacement of barium filled oesophagus by left auricle.

under Dr. Bramwell, and was given digitalis with improvement in her capacity for exertion. She remained under our observation, with persistent fibrillation, and, apart from the pain, relatively well, until August, 1945, when she was readmitted to hospital because her pain, hitherto tolerable, had become very severe and almost continuous, being no longer relieved by rest. A cardiogram six days before admission showed auricular fibrillation with right axis deviation (Fig. 3A).

On examination she was much wasted, and distressed by pain. The pulse was completely irregular, rate 136 a minute; blood pressure $130 / 90 \mathrm{~mm}$. The heart was enlarged; at the apex a very loud first sound and a long rumbling diastolic murmur were heard. There was no evidence of right ventricular failure. The right lower chest was dull on percussion and breath sounds and tactile fremitus were almost absent; moist sounds were present at both lung bases. Systolic retraction was conspicuous in the sixth, seventh, and eighth intercostal spaces in the right mid-axillary line. On cardioscopy the heart was grossly enlarged and the right lower chest was radio-opaque. Right oblique and lateral views failed to show any erosion of the spine; unfortunately left oblique views were not taken.

Two days after admission normal rhythm with a prolonged $\mathbf{P}-\mathbf{R}$ interval was recorded (Fig. 3B) and, apart from several attacks of regular tachycardia (Fig. 3C) lasting some hours, 

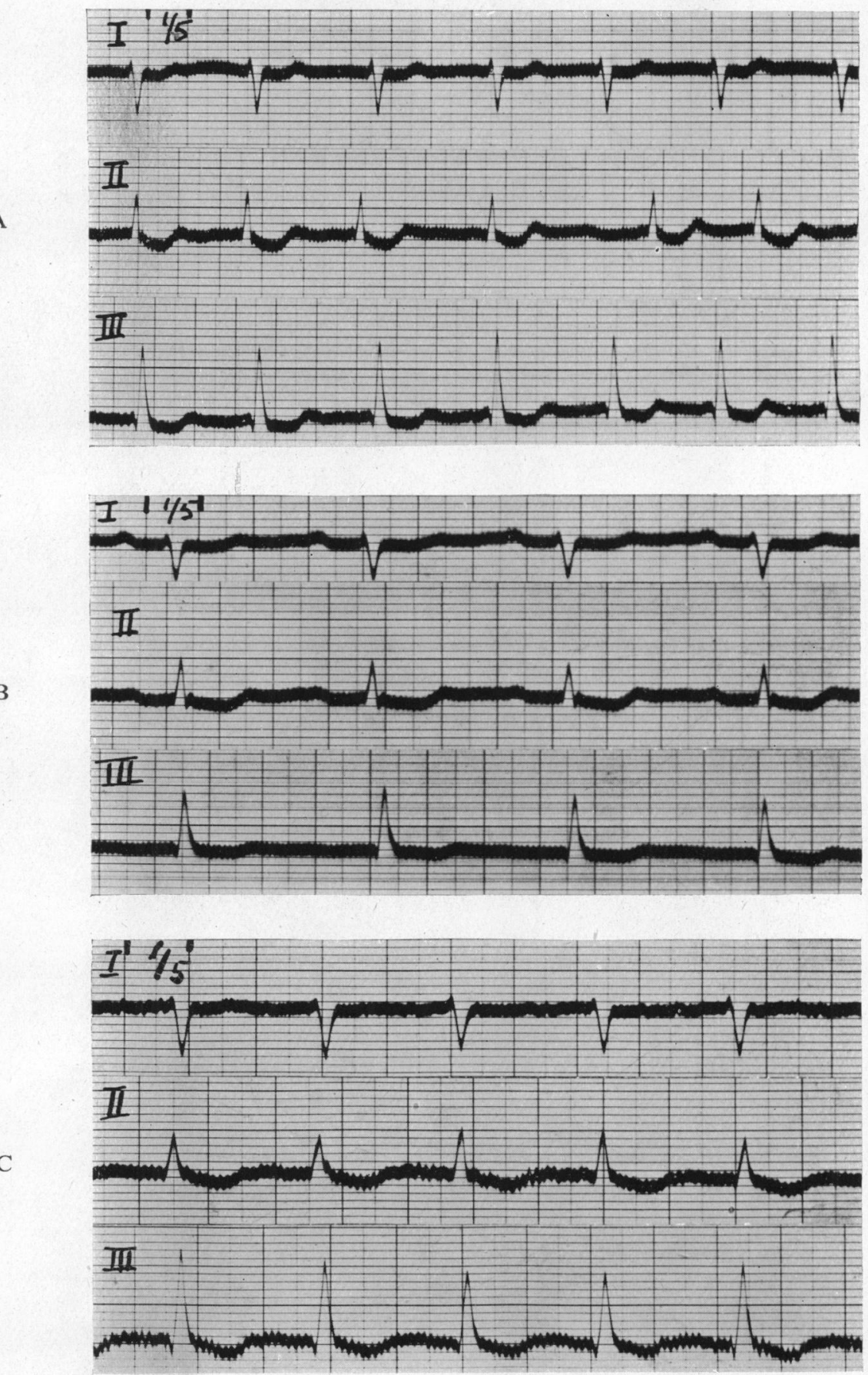

FIG. 3.-(A) Cardiogram six days before admission, showing auricular fibrillation with right axis deviation and digitalis effect.

(B) Cardiogram two days after admission, showing sinus rhythm with a prolonged P-R interval.

(C) Cardiogram during an attack of regular tachycardia, rate 140 a minute. (Calibration: $3 m V=$ $2 \mathrm{~cm}$.)

this persisted until her death seven weeks later. In spite of treatment her condition gradually deteriorated; she became more feeble and wasted, and her pain increased in severity, being relieved only for short periods by full doses of morphia. Two days before death slight oedema of the legs appeared, but there was no gross right ventricular failure.

Necropsy was performed by Dr. F. A. Langley, 23 hours after death. The heart weighed 


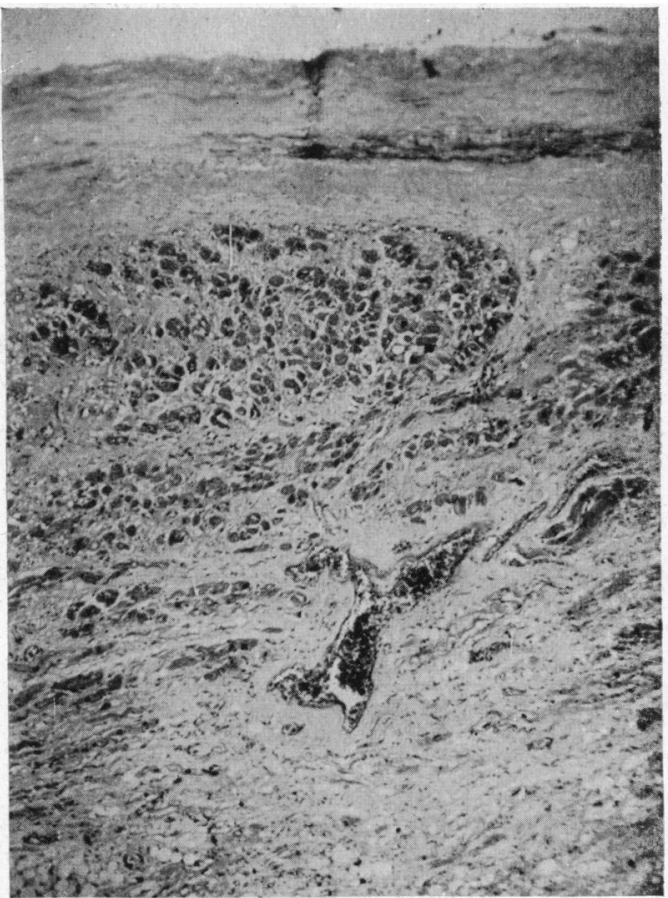

Fig. 4.-Section of left auricular wall stained with haemalum and eosin. Although there is some fibrosis, a considerable number of muscle fibre bundles can be seen. Magnification: $\times 36$.

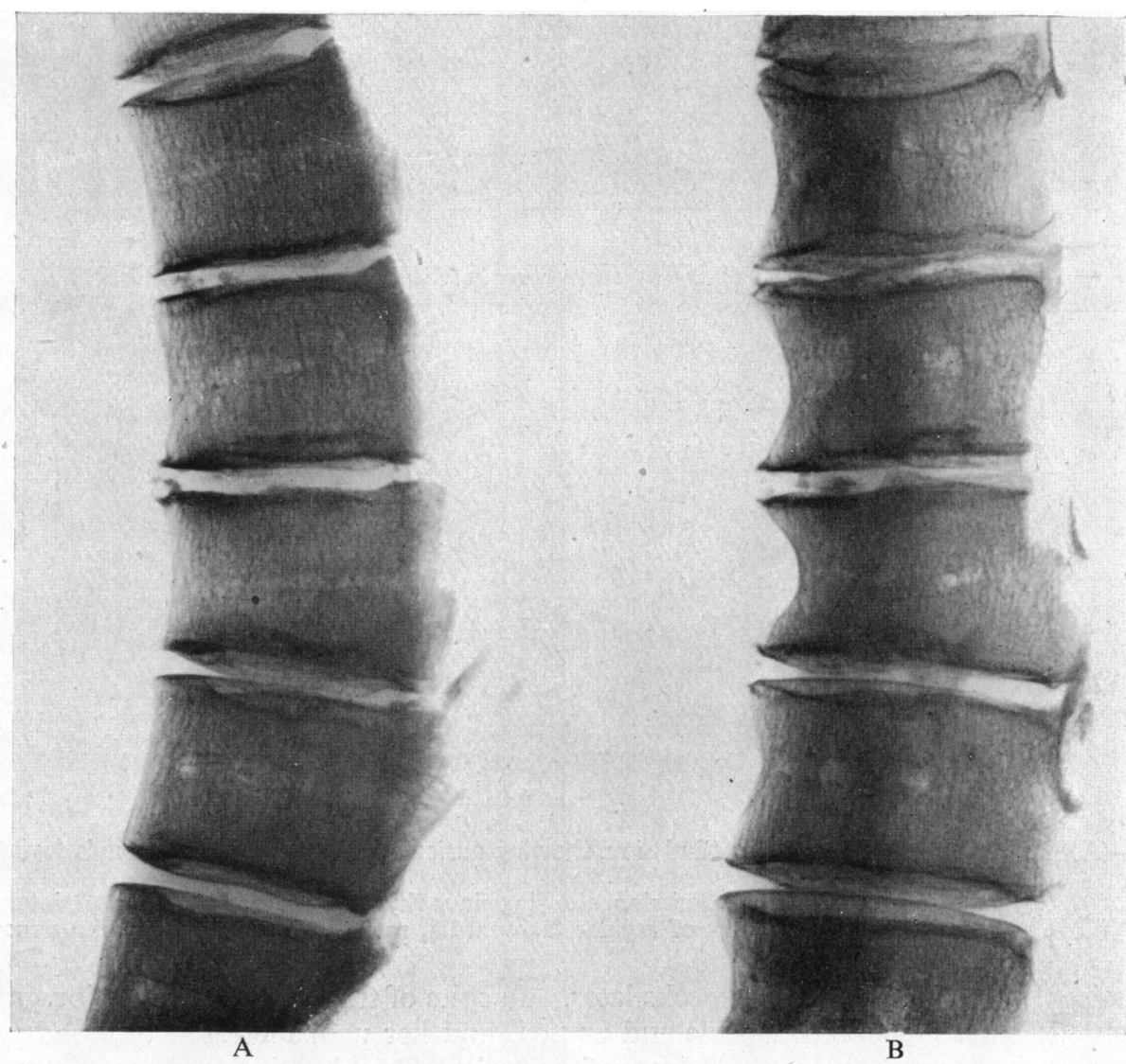

5.- Post-mortem radiograms of the dorsal spine. (A) Lateral view which fails to show the erosion. The deeply eroded vertebra is the seventh dorsal. 
$420 \mathrm{~g}$. The right border, formed by the left auricle, extended $10 \mathrm{~cm}$. to the right of the midline. The lower lobe of the right lung was partially collapsed and firmly adherent to the chest wall laterally and to the pericardium and left auricle medially. The left auricle was enormously dilated and its wall thin; microscopy of the wall revealed some fibrosis, but a considerable amount of muscle tissue was present (Fig. 4). The right auricle was moderately dilated; the right ventricle was $3-4 \mathrm{~mm}$. in thickness. The left ventricle was moderately dilated, the wall measuring $1 \mathrm{~cm}$. in thickness. The mitral valve was severely stenosed and reduced to a narrow slit $1.5 \mathrm{~cm}$. in length; the tricuspid valve was slightly stenosed (circumference $10.5 \mathrm{~cm}$.) and its cusps and chordæ tendinæ were thickened. The aortic cusps were slightly thickened; the pulmonary valve appeared healthy. The fifth, sixth, seventh, eighth, and ninth thoracic vertebral bodies were eroded, the intervertebral discs being spared. The erosion of the seventh vertebra was most conspicuous (Fig. 5). Apart from some chronic venous congestion, the remaining organs were healthy.

\section{Discussion}

Erosion of the spine by a dilated pulsating auricle lying against it does not seem surprising, but we have been unable to find any report of a similar case. Since importance has been attached to pulsation as a factor in the mechanism of erosion of bone by arterial aneurysms, it is interesting that a fibrillating auricle should be capable of similar erosion. It is tempting to suggest that reversion of the auricle to normal rhythm played a part in the exacerbation of the pain in the later stages. The presence of vertebral erosion combined with the distribution of pain strongly suggested root pressure, which would explain the intractable character of the pain. Had this possibility occurred to us during life, paravertebral'root injection might have been tried, for there seems little doubt that the severe continuous pain played an important part in the progressive deterioration of the patient's condition that ultimately led to death. In the earlier stages pain was provoked by exertion and relieved by rest. It is possible that the rise of venous pressure during exertion, by further distending the auricle, increased root pressure, and that the fall in auricular pressure with rest reduced it. Pain in the right chest on exertion in a case of aneurysmal dilatation of the left auricle was described by Bedford (1927) but necropsy findings were not available. Systolic pulsation on the right side of the chest has been recorded by Dressler (1937) and by Bedford (1927) but we have not found a case in which systolic retraction was noted.

It seems surprising that, after years of fibrillation, the enormously dilated left auricle should have been capable of reverting spontaneously to normal rhythm. We have, however, seen normal rhythm return in a case of greatly dilated left auricle in which fibrillation had persisted for 19 years; but in that case fibrillation recurred within a week. The amount of muscle tissue in the auricular wall in the present case was much greater than that usually found in an extremely dilated left auricle and this may have facilitated reversion to normal rhythm.

\section{SUMMARY}

A case of aneurysmal dilatation of the left auricle associated with mitral stenosis is described in which severe pain in the chest was a prominent symptom. Necropsy showed erosion of the bodies of several dorsal vertebrae, due to pressure from the dilated auricle. Systolic retraction of the chest wall in the right axilla and spontaneous reversion to normal rhythm after fibrillation had persisted for several years were other unusual features of the case.

We are indebted to Professor Crighton Bramwell for permission to publish details of this case, to Dr. E. Duff Gray for the clinical radiograms, to Dr. F. A. Langley for his help with the pathology, and to Mr. F. Ward for the post-mortem radiograms and the microphotograph.

\section{REFERENCES}

Bedford, D. E. (1927). Amer. Heart J., 2, 127.

Dressler, W. (1937). Arch. intern. Med., 60, 663. 